\title{
HARTNUP DISEASE IN PSYCHIATRIC PRACTICE: CLINICAL AND BIOCHEMICAL FEATURES OF THREE CASES
}

\author{
BY \\ L. A. HERSOV* and R. RODNIGHT
}

From the Maudsley Hospital, London

In a previous paper the case history was given of a 10-year-old boy admitted to hospital for a psychotic illness associated with a photosensitive skin rash (Hersov, 1955). The condition resembled classical pellagra, and although no dietary reason for a vitamin deficiency was apparent, a good response was obtained to treatment with nicotinamide. Biochemical investigations revealed gross and persistent indicanuria and it was suggested by Rodnight and McIlwain (1955), who studied this aspect of the illness, that the nicotinamide deficiency might be due to a diversion of trytophan from its normal route of nicotinamide formation into that of indican formation.

Shortly after the publication of these papers we were approached by Professor C. E. Dent, who expressed interest in the patient and with whose help we were able to show that the boy was an instance of Hartnup disease, the hereditary pellagra-like disorder later reported in detail by Baron, Dent, Harris, Hart, and Jepson (1956). The syndrome described by these workers occurred in four out of eight siblings of a marriage between first cousins and was characterized by attacks of dermatitis and cerebellar ataxia; it was also claimed that the condition was probably associated with progressive mental deterioration. Well-defined biochemical abnormalities were present in the affected siblings, consisting of a constant renal aminoaciduria of distinctive pattern and a high excretion of indican and other abnormal tryptophan metabolites. Our patient was shown to have an aminoaciduria of exactly the same pattern, and although his clinical symptoms were in some respects different, there is no doubt that he has the same basic disorder.

We were stimulated by this observation to search for further cases of Hartnup disease amongst psychiatric patients. A female patient with similar symptoms, kindly brought to our notice by Dr. Denis Leigh, was then found to have the biochemical

* Present address: Child Guidance Training Centre, Osnaburgh Street, London. abnormality and examination of her family revealed an affected sister. The present paper describes the follow-up of our original case for six years, together with as much information on his family as it has been possible to obtain, and the clinical and biochemical features of the second family. A preliminary report of some of the biochemical data has already been given (Rodnight, 1959a).

\section{Case Reports}

Family A.-This is the family of the boy (M.H.) whose first attack of Hartnup disease has already been described by one of us (Hersov, 1955). The parents are not related. The incidence of amino-aciduria in the family is given in Table I. M.H. remains the only affected member, but it $\stackrel{\mathbb{Q}}{\mathcal{D}}$ has unfortunately been impossible to test the father (whog deserted some years ago) and a brother on military service in the Far East. Further relatives have been sought without success.

M.H. was admitted to the Children's Department of the Maudsley Hospital in May, 1953, and discharged free? of symptoms in July of that year. Shortly afterwards the family moved to the north of England and contact with the patient has been maintained through his school medical officer and general practitioner. He ceased taking extra nicotinamide (prescribed on discharge) in October, 1955, and apart from a short spell of diarrhoea in the summer of 1954 and a transient skin rash in the spring of 1957, he has remained in good health. He completed schooling at a secondary modern school without difficulty and is now apprenticed to a transport corporation as a mechanic. The last report we had from his general practitioner (Dr. C. Potts) was in April, 1958, when no skin lesions or neurological or psychiatric abnormalities were found.

Family B.-The parents are not related and there are three daughters; the incidence of aminoaciduria is shown in Table I. The second daughter (E.) was first treated at the age of 18 years in 1949 by Dr. Denis Leigh for a psychiatric disorder associated with pellargrous skin lesions. Following publication of the paper by Baron et al. (1956) we tested her urine for amino-acids and found her to be a case of Hartnup disease. Investigation of her family then showed that the eldest sister (J.) was also suffering from the disease, but there were no other affected members. 
TABLE I

INCIDENCE OF AMINO-ACIDURIA IN THE FAMILIES

\begin{tabular}{l|c|l}
\hline \multicolumn{1}{c|}{ Subject } & Age (years) & Aminoaciduria \\
\hline $\begin{array}{l}\text { Family } A \\
\text { Fatner } \\
\text { Mother } \\
\text { M., female }\end{array}$ & - & Not tested \\
R., male & - & Absent \\
S.,female & 28 & Absent \\
M., male & 26 & Not tested \\
Family B & 23 & Absent \\
Father & 16 & Present \\
Mother & & \\
J., female & - & Absent \\
E., female & - & Absent \\
B., female & 29 & Present \\
\hline
\end{tabular}

$E$. - The patient is now a married woman of 27 years. She was a healthy child apart from enuresis and tension habits which ceased at the age of 6 years. Her parents noticed from an early age that her skin would easily redden and "peel" after exposure to sunlight. A severe attack occurred during a summer holiday when she was 8 years old, the exposed areas of her body becoming red, painful, and later dark and scaly. There was another severe recurrence of the same skin lesions in the summer of 1948, the year before her admission to hospital.

She was a rather timid, dependent child, attended a central school, and was generally average in class with no behaviour problems. She began work as a duplicating machine operator and continued in this occupation until she fell ill. At work she was popular, sociable, able, and easy to supervise.

In October, 1948, she gradually experienced weakness and stiffness in the lower limbs and on several occasions "collapsed" while walking and had to be assisted. She was able to continue work until February, 1949, when she complained of giddiness and double vision. This was followed by complaints of recurrent feelings of stiffness in the back and legs, which felt numb and tremulous after exertion. She gave up work, but failed to improve. In April, 1949, she became severely depressed with suicidal preoccupation and complaints of inability to feel properly, and was admitted to the Maudsley Hospital.

Physical examination showed a fair-skinned, wellnourished girl ( $7 \mathrm{st} .7 \mathrm{lb}$.) with no skin lesions or abnormality in the central nervous system apart from brisk reflexes. All other systems were normal. She was untidy in appearance, depressed, apprehensive, tense and tearful, expressing suicidal ideas. She described severe and persistent feelings of depersonalization. No hallucinations or delusions were elicited; orientation, concentration, and memory were unimpaired. Her state showed no change during the following three weeks: she remained depressed and depersonalized, slept poorly, and did not participate in ward activities, complaining of stiffness and weakness in her limbs; at times she required assistance when walking. Then, after exposure to sunlight, she developed a severe erythema of the exposed skin areas, together with an exacerbation of the depression and depersonalization. No abnormality of porphyrin metabolism was found in the urine, and her history and clinical picture led to a diagnosis of pellagra. Treatment with oral nicotinic acid (200 mg. daily) resulted in the patient becoming markedly more cheerful and active after 72 hours, followed by a decrease in the depression and depersonalization. Within a week the skin lesions had returned to normal, and she was also free of psychiatric symptoms and somatic complaints. Prolonged exposure to sunlight then had no further harmful effect and she was discharged home fully recovered in July, 1949, after three months in hospital.

The patient has been followed up as an out-patient over the past 10 years. In May, 1950, she suffered from weekly fainting attacks which came on without warning and lasted one or two minutes. There was no tongue biting, incontinence, or muscular spasms, and no drowsiness or confusion followed the attacks. Enquiry revealed no history of previous epileptic attacks and no family history of epilepsy. However, an electroencephalogram at this time showed a number of non-specific anomalies at varying frequencies of photic stimulation; coincidental injection of "metrazol" elicited a short burst of bilaterally synchronous 3 per second spike and wave. The fainting attacks ceased with the administration of nicotinic acid (100 mg. daily). The patient remained well throughout the summer of that year and did not develop any skin lesions. Nicotinic acid was discontinued in October, 1950.

In June, 1951, she had a similar fainting attack on one occasion, but again with no skin lesions or other symptoms apart from irregular menstrual periods. Nicotinic acid was again prescribed and continued throughout the winter. In the summer of 1952 she again had fainting attacks once a week for four weeks, but they ceased when "phenytoin" (grains thrice daily $1 \cdot 5$ ) and nicotinic acid (75 mg. daily) were given.

In June, 1953, the patient developed a sore tongue, angular stomatitis, and an erythematous scaly dermatitis of the exposed areas of the face and hands. There were no complaints of fainting, depression, or depersonalization and the skin lesions responded rapidly to treatment with nicotinic acid.

Since then the patient has remained free of symptoms apart from mild anxiety and tension lasting a few months and related to marital difficulties. A neurological and psychiatric examination in November, 1958, showed no abnormality. On this occasion psychological tests were also carried out using the Wechsler intelligence scale with the following results: full-scale I.Q., 95; verbal I.Q., 102; performance I.Q., 88. The patient is now in regular employment as a shop assistant.

$J$.- The patient, a single woman of 29 years, is an experienced typist holding a responsible position. She is a reticent, unassertive person with few friends and limited interests. When 8 years old she suffered from severe osteomyelitis of the left leg, requiring operative treatment, 18 months in hospital, and a caliper until she was 16 years old. While convalescing from the operation she remembers developing severe sunburn when exposed to the sun, requiring treatment with lotions and protective masks. Because of this liability to sunburn she eventually had to be confined indoors. Since then she has been careful to avoid excessive exposure to direct sunlight and protects her skin with lotions. This symptom has not reappeared. 
In 1948, at the age of 18 years, she was referred to the medical out-patient department of a general hospital for "collapsing attacks" and backache. The symptoms were of sudden onset and increased in severity over a period of six months. After the "collapsing attacks" her legs would feel stiff and weak, and she would be unable to resume walking without a period of rest. During these times she was also unusually tense, anxious, tearful, and irritable; she was unable to work. She was referred to a psychiatrist, who made a diagnosis of "anxiety state" and prescribed a holiday of four weeks, during which her symptoms disappeared completely. She has remained well since with no further symptoms. She was last examined at the Maudsley Hospital in November, 1958, when no neurological or psychiatric abnormalities were found. An electroencephalogram at this time, however, showed a generalized abnormality with an excess of central theta rhythm, but no specific epileptic features. Psychological testing on the Wechsler intelligence scale gave an I.Q. of 114 .

Investigation of Family.-The father, aged 63 years, has been treated in the past for a duodenal ulcer but has no history of skin lesions, or of neurological or psychiatric illnesses. The mother, aged 63 years, is a normal and healthy person. Both parents come from large families, the majority of whose members have emigrated or cannot be traced.

The youngest daughter (B.) is a single girl (26 years) of a healthy, stable personality and above average intelligence (Wechsler intelligence scale I.Q. 118). There is nothing unusual in her medical history. Two maternal nieces are backward children: the older is feeble-minded (I.Q. 60), but it has not been possible to test her urine; the younger is an imbecile and has been tested for aminoaciduria with a negative result.

\section{Biochemical Methods}

Paper Chromatography.-Two-dimensional paper chromatography was carried out on $9 \times 9$ in. squares of Whatman No. 20 paper in the apparatus described by Rodnight and Aves (1958).

Urinary Amino-acids. - The determinations were made by paper chromatography. Urine specimens were electrolytically desalted and a series of two-dimensional chromatograms were prepared using volumes of urine containing from 10 to $75 \mu \mathrm{g}$. nitrogen, in parallel with an appropriate range of standards. The solvents used were mixtures of butanol and acetic acid and phenol and ammonia (Smith, 1958). Amino-acids were revealed by ninhydrin and the intensities of the spots matched visually against the standards after storing the papers in the dark for 24 hours. Several determinations at different levels were made for each specimen and the maximum range of error estimated as $25 \%$. Tryptophan was measured separately by a method given below. Total amino-acid nitrogen was determined by formol titration; since desalted samples were used no correction for urinary ammonia was required.

Urinary Indoles.-Indole chromatograms of fresh urine specimens were prepared according to Rodnight and Aves (1958). Tryptophan was separated on de- activated charcoal (Asatoor and Dalgliesh, 1956) and determined on two-dimensional chromatograms by visual comparison with a range of standards. Serotonin and tryptamine were determined by the method of Rodnight (1956), except that the "amberlite" resin CG-50 was used in place of "zeo-karb" 226 and the final estimations were made on two-dimensional chromatograms. Indole acids were determined by a chromatographic method described elsewhere (Rodnight, 1959b). Indican was determined according to Sharlit (1933).

Whole blood indoles were examined by an acetone extraction procedure described elsewhere (Rodnight, 1959b).

Urinary Kynurenic Acid and Xanthurenic Acid.-The fluorometric method of Satoh and Price (1958) was used. Fluorescence was measured in a Locarte fluorometer using the following filters for the primary and secondary sides of the instrument respectively. Co.).

Kynurenic Acid.-OX7 (Chance Bros.); LF3 (Locarte

Xanthurenic Acid.--OV1; GR2 and OY3 (all Chance Bros.).

Recovery of these substances added to urine ranged from 65 to $90 \%$; internal standards were therefore used in each estimation and the results quoted are corrected for losses.

Results of Biochemical Investigation

Aminoaciduria.-The incidence of this in the two families has already been given (Table I). The affected members in both families have been tested $\stackrel{\mathbb{Q}}{\varrho}$ at approximately yearly intervals over a period of six years in the case of $M$. in family $A$, and over three years for cases E. and J. in family B. Theo highly distinctive pattern of aminoaciduria was present on each occasion, and only minor differences could be discerned between the subjects by visual inspection of the chromatograms.

In order to determine the magnitude of the loss of amino-acids in our cases, estimates were made of the daily output of total amino-acid nitrogen and of the individual amino-acids in family B (Table II).

TABLE II

DAILY URINARY EXCRETION OF AMINO-ACIDS IN FAMILY B

\begin{tabular}{l|c|c|c}
\hline \multicolumn{1}{c|}{ Amino-acid } & J. & E. & B. \\
\hline Alanine & 650 & 500 & 50 \\
Asparagine & 500 & 330 & Not detected \\
Aspartic acid & Not detected & 160 & Not detected \\
Glutamic acid & 430 & 40 & Not detected \\
Glutamine & 3,200 & 2,500 & 320 \\
Glycine & 650 & 1,000 & 150 \\
Histidine & 650 & 500 & 430 \\
Leucine & 550 & 330 & Not detected \\
Isoleucine & 430 & 500 & Not detected \\
Lysine & 210 & 250 & Not detected \\
Phenylalanine & 1,300 & 1,000 & 100 \\
Serine & 500 & 500 & Not detected \\
Threonine & 40 & 40 & 15 \\
Tryptophan & 500 & 330 & 30 \\
Tyrosine & 650 & 330 & Not detected \\
Valine & 1,290 & 1,099 & 151 \\
Total amino-acid N & & & \\
\hline
\end{tabular}

Results expressed in mg. per 24 hours. 
Amino-acid excretion in subjects $\mathbf{J}$. and E. exceeded that in the normal sister B. by a factor of nearly 10 , and variable increases were found in all the aminoacids, with the exception of histidine, where the difference lay within the error of the method. Especially notable is the excretion in the affected cases of 2 to $3 \mathrm{~g}$./day of glutamine and over $1 \mathrm{~g}$. of serine. With minor differences, therefore, the urinary amino-acid pattern in these cases closely resembles that described by Baron et al. (1956) and Evered (1956) in the original Hartnup family, although in the latter study glutamine was apparently measured as glutamic acid.

Indoles.-Baron et al. (1956) and Jepson (1956) reported an abnormally high excretion in members of the Hartnup family of four indoles, namely tryptophan, indican, indolylacetic acid, and indolylacetylglutamine. This pattern has been reproduced in $\mathbf{M}$. of family $\mathbf{A}$ on each occasion his urine has been tested (by two-dimensional paper chromatography) over a period of six years. J. and E. in family B have been examined on nine occasions over three years with a rather different result: thus, although the level of indican was consistently raised in all specimens, tryptophan was generally only moderately raised, whilst indolylacetic acid and indolylacetylglutamine were within normal limits in all specimens.

Several specimens from all three cases contained an unknown indole not described by Baron et al. (1956) or by Jepson (1956) in the Hartnup family cases. On two-dimensional chromatograms this occupies a position roughly between the origin and the urea spot (Rodnight and Aves, 1958); the purple appears very rapidly after the application of Ehrlich's reagent and fades to invisibility within three minutes. Its nature has not yet been investigated.

Quantitative estimates of the daily output of some urinary indoles, including those mentioned above, are quoted in Table III for Case M. of family A and for the three siblings of family $B$. Indican excretion

TABLE III

DAILY URINARY EXCRETION OF INDOLES

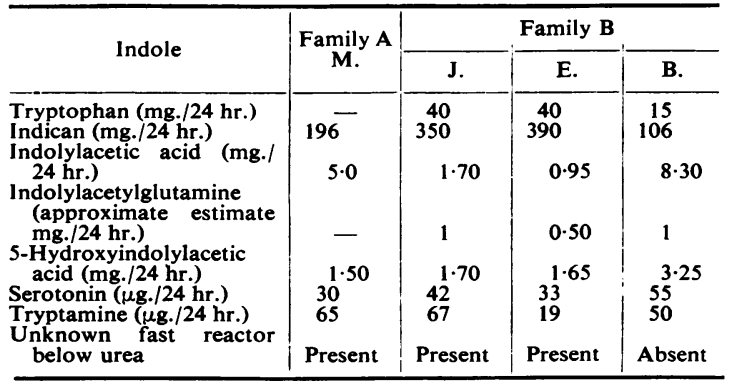

is markedly raised in Cases $J$. and E., high but almost within normal range in Case $M$., and normal in the unaffected subject $\mathrm{B}$. Confirming the impression gained from the qualitative chromatography, the outputs of indolylacetic acid and indolylacetylglutamine are normal in all the affected cases; the rather high value for indolylacetic acid obtained from the normal sister B. was also noted in two other specimens from this subject.

The other urinary indoles measured were serotonin, its principal metabolite in the body (5-hydroxyindolylacetic acid) and tryptamine; the latter is a possible precursor of indolylacetic acid. The output of serotonin tended to be low in the affected cases, but the values lie just within the normal limits of 30 to $120 \mu \mathrm{g}$. $\pm 20 \%$ per day as established at present (Rodnight, 1956, 1959b). A rather lower output of only $20 \mu \mathrm{g}$./day was obtained on another 24-hour sample from Case E. With regard to the excretion of 5-hydroxyindolylacetic acid, the values quoted in Table III for the affected cases are all below the lower limit of the normal range, but that for the normal subject $B$. may be considered as normal. Tryptamine output was normal in all the subjects.

Whole blood indoles were examined on one occasion in the two affected members of family B. No qualitative differences were detected from the pattern previously established in normal subjects (Rodnight, 1959b).

Urinary Kynurenic Acid and Xanthurenic Acid.These metabolites were determined as they lie on the pathway from tryptophan to nicotinamide. The results of estimates made on the 24-hour samples from family B. are quoted in Table IV together with the 24-hour outputs of several normal subjects, since few normal values using the fluorometric method have been published as yet. The output of kynurenic acid in the affected subjects is definitely lower than in the normal sister or in the other normal subjects; a similar difference, though less marked, is also apparent in output of xanthurenic acid.

TABLE IV

\begin{tabular}{l|c|c} 
URINARY KYNURENIC AND XANTHURENIC ACIDS \\
\hline Subjects & $\begin{array}{c}\text { Kynurenic } \\
\text { Acid } \\
\text { (mg./24 hr.) }\end{array}$ & $\begin{array}{c}\text { Xanthurenic } \\
\text { Acid } \\
\text { (mg./24 hr.) }\end{array}$ \\
\hline $\begin{array}{c}\text { Family } B \\
\text { J. }\end{array}$ & 0.74 & 1.75 \\
E. & 0.66 & 1.54 \\
B. & 2.10 & 2.25 \\
Normal subjects & & \\
Male, 23 yr. & 2.28 & 2.15 \\
Male, 24 yr. & 2.68 & 1.66 \\
Male, 26 yr. & 3.85 & 3.25 \\
Female, 23 yr. & 3.95 & 2.60 \\
Female, 24 yr. & 3.80 & 5.10 \\
\hline
\end{tabular}




\section{Discussion}

All suspected cases of Hartnup disease at the Maudsley Hospital over the past four years have been carefully screened for indicanuria and aminoaciduria, with negative results except for the new cases reported here. In an earlier study no case of severe indicanuria had been found amongst 50 unselected new admissions to another mental hospital (Rodnight and Mcllwain, unpublished data) nor were any instances of the Hartnup indole pattern detected in a more recent study of indole excretion in 156 unselected mental hospital patients (Rodnight and Aves, 1958). Further cases of the condition reported by other authors since the original account of Baron et al. (1956) comprise three children described by Jonxis (1957) members of two different consanguineous families in Holland, a 4-year-old girl reported by Henderson (1958), and a 7-year-old girl reported by Weyers and Bickel (1958) from Germany. Thus, so far as we are aware, the present account brings the total number of published instances of the condition up to 13 cases occurring in eight families, three of which are consanguineous. The condition therefore appears a very rare one, but we agree with Baron et al. (1956) in considering that some caution is still necessary before accepting this conclusion, particularly since the clinical manifestation of the disease has now been shown to be quite variable and often relatively mild in character, so that minor attacks in subjects with the biochemical defect may well go undetected or undiagnosed. Moreover, it now appears that attacks of the disease may only occur very rarely, as exemplified by the present patients, all of whom have suffered only one major attack, which in J. and E. of family B was delayed until early adult life.

In the previous accounts of Hartnup disease cited above, the psychiatric features of the clinical picture have ranged from emotional lability in the milder cases to apathy, irritability, depression, confusion, and delirium in the more severe cases. In the earlier cases, however, these symptoms were apparently preceded and overshadowed by neurological and other features, since in no case were they the primary cause of referral to hospital. In the attacks suffered by our patients, psychiatric symptoms were a prominent clinical feature, taking the form of hallucinations and delusions in the boy (Case M., Hersov, 1955), depression and depersonalization in Case E., and an anxiety reaction in Case J. In classical pellagra, also, the presenting symptoms may vary considerably, some subjects exhibiting psychiatric disturbances to a greater extent than others (Bicknell and Prescott, 1953).

The psychiatric symptoms occurring in Hartnup disease appear to be non-specific reactions, which respond well to treatment and leave no residual effects. Hallucinations and delusions are wellknown accompaniments of toxic and infectious conditions in childhood (Kanner, 1948). Depersonalization has been described by Mayer-Gross (1935) in organic cerebral disease and in normal subjects in states of fatigue; he views it as a preformed functional response of the brain in the same category as other non-specific preformed functional mechanisms such as epileptic fits, delirium, states of semi-consciousness, and catatonic states.

The suggestion was made by Baron et al. (1956) that the biochemical defect in Hartnup disease may slowly cause progressive mental deterioration. Our own data give no support to this suggestion. The I.Q.s of our patients were all within the average range, and Case E. in fact showed a raised I.Q. when retested eight years after her first attack (the scores of the progressive matrices for 1949 and 1958 were I.Q. 94 and I.Q. 107 respectively). Nor has any decline in school progress or work record been observed in our patients over a long period of time. The conclusion of Baron et al. (1956) was based mainly on their finding that the I.Q.s of the four affected children in their family diminished from a value of 101 in the youngest to 61 in the eldest, and also on the mother's report of an apparent deterioration in behaviour as they grew older with the appearance of bullying attitudes and disagreeable moods. Such behaviour is commonly seen in children and adolescents as a reaction to the handicap of illness, periods in hospital, and educational backwardness, and is in no way comparable with deterioration as commonly defined: a progressive change in the direction of decreasing efficiency in the performance of certain tasks as compared with past performance. Similarly the decline in I.Q. with age might also be ascribed with equal justification to detrimental environmental influences as to genuine deterioration, since the I.Q.s in the affected children were lowest in whom the attacks of the disease had been most frequent and severe, and highest in cases with mild symptoms or no symptoms. In our view, therefore, the psychological evidence of Baron et al. (1956) is inadequate to support the conclusion that a progressive decline in intellect occurs in Hartnup disease, particularly since it fails to take into account the effects of illness and repeated periods in hospital on I.Q. scores and social skills.

The biochemical observations on the present cases mainly confirm the work of Baron et al. (1956) and Jepson (1956); in particular they emphasize the remarkable uniformity of the defect in aminoacid excretion. With regard to the indoles, it is now clear both from the present work and from that of Milne, Crawford, Girao, and Loughridge (1959) 
that a raised excretion of indolylacetic acid and indolylacetylglutamine is not a constant characteristic of the disease. In our cases, however, indican excretion has always been high, and in the absence of facilities for preparing amino-acid chromatograms determinations of indican may serve as a useful guide in suspected cases, although the final diagnosis must rest on the demonstration of the typical aminoaciduria. The excretion of serotonin has not been previously investigated in Hartnup disease, but it appeared possible that this important pathway of tryptophan metabolism, as well as the route to nicotinamide, might also be affected by the distortion of tryptophan utilization which occurs in the disorder. In fact no major abnormality was found in the present study, but the daily output of 5-hydroxyindolylacetic acid and to a lesser extent that of serotonin itself, tended to be lower than normal in the affected subjects. Although more results are necessary to confirm this tendency, the present figures suggest that some diversion of tryptophan from the pathway of serotonin formation may occur in the disease. Such a diversion would be consistent with the suggestion of Milne et al. (1959) that the distortion of tryptophan metabolism may be due to a defect in the transport of tryptophan rather than to a deficiency of tryptophan peroxidase. However, in phenylketonuria, another hereditary disease, an established enzyme deficiency is associated with a deficiency in serotonin (Pare, Sandler, and Stacey, 1957).

Low excretion rates were also obtained for kynurenic acid, a result which confirms the observation of Milne et al. (1959) that Hartnup subjects metabolize an abnormally low percentage of ingested tryptophan to kynurenine, the precursor of kynurenic acid.

\section{Summary}

The clinical and biochemical features are described of three cases of Hartnup disease presenting with non-specific psychiatric disorders. No evidence for mental retardation or deterioration was found in the patients.

Generally normal values were found for the excretion of indolylacetic acid and indolylacetylglutamine, but in conformity with previous work the excretion rate of indican in the patients was consistently raised. Low excretion rates were found for serotonin, 5-hydroxyindolylacetic acid, and kynurenic acid.

We wish to express thanks to our colleagues for their help in this study. In particular we are very grateful to Professor H. Mcllwain for his encouragement and advice throughout the work, to Dr. Denis Leigh for permission to use his case records and for much helpful advice, and to Dr. C. Potts for cooperation in following up one of the subjects. Mr. E. K. Aves rendered excellent technical assistance. The work was assisted by a grant from the Research Fund of the Bethlem Royal and the Maudsley Hospital, which we acknowledge with thanks.

\section{REFERENCES}

Asatoor, A., and Dalgliesh. C. E. (1956). J. chem. Soc., p. 2291. Baron, D. N., Dent, C. E., Harris, H., Hart, E. W., and Jepson, J. B. (1956). Lancet, 2, 421.

Bicknell, F., and Prescott, F. (1953). The Vitamins in Medicine, 3rd ed., p. 354. Heinemann, London.

Evered, D. F.'(1956). Biochem. J., 62, 416.

Evered, D. F. (1956). Biochem. J., 62, 416.
Henderson, W. (1958). Arch. Dis. Childh. 33, 114.

Henderson, W. (1958). Arch. Dis. Childh. 33,
Hersov, L. A. (1955). J. ment. Sci., 101, 878.

Jepson, J. B. (1956). Biochem. J., 64, 14P.

Jonxis, J. H. P. (1957). Ned. T. Geneesk., 101, 569.

Kanner, L. (1948). Child Psychiatry, 2nd ed., p. $449 . \quad$ Thomas, Springfield, Illinois.

Mayer-Gross, W. (1935). Brit. J. med. Psychol., 15, 103.

Milne. M. D., Crawford, M. A., Girao, C. B., and Loughridge, L. (1959)., Biochem. J., 72, 30 .

Pare. C. M. B., Sandler, M., and Stacey, R. S. (1957). Lancet, 1, 551 .

Rodnight, R. (1956). Biochem. J., 64, 621.

(1959a). Proceedings of Third International Neurochemical Congress, Strasbourg.

(1959b). Ph.D. Thesis. London University.

, and Aves, E. K. (1958). J. ment. Sci., $104,1149$.

C, and Mc!lwain, H. (1955). Ibid., 101, 884 .

Saton. K., and Price, J. M. (1958). J. biol. Chem., 230, 781.

Sharlit, H. (1933). Ibid., 99, 537.

Smith, I. (1958). Chromatographic Techniques. Heinemann, London.

Weyers, H., and Bickel, H. (1958). Klin. Wschr., 36, 893. 